\title{
Evolution and future directions for the concept of mild cognitive impairment
}

The term mild cognitive impairment has been associated with a varying degree of clinical utility and controversy. The concept has been introduced to try and define a pre-dementia period associated with underlying neurodegenerative pathology and a higher likelihood of the person developing a dementia syndrome. As scientific understanding improves then the definition of MCI rightly adapts, meaning that the MCI concept is prone quite rightly to frequent evolution. We consider that we are a long way away from the concept having evolved to a point where it can be embedded with confidence in clinical practice as a diagnosis but should remain as a term primarily for use in research.

\section{A move away from a formerly categorical approach to a clinical spectrum}

The origins of term mild cognitive impairment (MCI) lie in a Canadian care home in the 1950's where it was used to group the home's residents to provide better, appropriate care (Heinik, 2006). Then, the term permeated into the research and clinical communities becoming a tool to categorize individuals. Today, however, there is a move away from a binary approach where individuals with mild memory problems are either considered to have or not have MCI. This shift is rooted both in the limited understanding of the developmental pathways of cognitive impairment as well as limitations in the assessment of what the contrast between normal versus impaired would be. MCI is a state between healthy aging and a diagnosis of dementia. While dementia manifests in a complex clinical presentation both in the biological changes and the functional deficits, with the distinctive feature being memory loss, the criteria of MCI have so far focused primarily on the memory component leaving aside other crucial components commonly identified in neurodegenerative disorders pathology. As such, it remains uncertain in our current definitions of MCI how the subtle functional changes of MCI should be measured and interpreted. By definition, the first symptomatic stage of MCI on the neurodegenerative disorders clinical spectrum is cognitive decline and if the decline progresses it would eventually lead to functional impairment at which point a dementia syndrome could be diagnosed. However, a clinician in assessing whether an individual's function is impaired inevitably involves a significant amount of subjectivity given the complex interaction between cognition, function and environment, and the clinicians' own experience and standards. The criteria for this judgment of what is expected of someone their age (and social and cultural background) are ill-defined and frustrated by the challenges of measuring objectively such a complex concept as a function. It seems more appropriate to view the approach to MCI in the context of the whole life course. Situating MCI on a spectrum with two constituent factors (1) cognition and the highly correlated function and (2) biology rather than a discrete category may - in light of recent very large disease modeling cohorts - aid in predicting decline to more advanced clinical manifestations of neurodegenerative disease. Moreover, these models will be enhanced by two other factors e.g. (3) change over time and (4) risk factors (both fixed and modifiable) to create what we have previously referred to as the four-dimensional probability spectrum (Ritchie et al., 2016)

\section{Biomarker testing to improve diagnostic accuracy}

For decades, MCI was defined primarily by cognitive complaints. However, viewing MCI as a part of a spectrum as opposed to a categorical approach would allow more accurate identification of and intervention for individuals who truly have a mild cognitive complaint due to neurodegenerative disease and who are consequently at a high risk of progressing into more advanced manifestations of e.g. Alzheimer's disease (AD). Since Ritchie and Ritchie's (2012) previous editorial, the major shift in the categorization of MCI has been the introduction of biomarkers to inform the identification - if not yet the prognosis - of $\mathrm{AD}$ being the underlying pathological driver of the symptoms. In 2011, the updated National Institute on Aging-Alzheimer's 
Association (NIA-AA) criteria for AD incorporated a period of preclinical $\mathrm{AD}$, when $\mathrm{AD}$ was present in the brain (and could theoretically be identified using biomarkers) but without clinical symptoms (Sperling et al., 2011). This allows us to focus on an at-risk patient group who are cognitively healthy with accordingly no functional detriment but who are developing brain changes that are consistent with an underlying $\mathrm{AD}$ pathology.

\section{Isolated and single biomarker validity remains poor}

Using biomarkers in case identification, or indeed for prognosis of the condition, should be carried out with appropriate caution and consideration accounting for what we know empirically about the value of the tests. MRI and/or PET are used increasingly in the assessment of cognitive impairment suspected of being due to neurodegenerative disease but it is not clear which of these yield the most accurate results (Kogan and Jeong, 2017). For fluid biomarkers, a recent review concluded a high sensitivity of the core biomarkers of neurodegeneration for $\mathrm{AD}$ and MCI due to AD (Olsson et al., 2016) though looking at the biomarker validity more closely, four Cochrane Diagnostic Test Accuracy reviews demonstrated that amyloid- $\beta$ levels or the CSF amyloid- $\beta /$ tau ratio whether measured in CSF (Ritchie et al., 2014; Ritchie et al., 2017) or using PET imaging (Zhang et al., 2014; Smailagic et al., 2015), had much lower specificity than sensitivity for people with MCI subsequently developing Alzheimer's dementia. This suggests that while measuring amyloid- $\beta$ levels is useful in identifying individuals who are at higher risk of transitioning into Alzheimer's dementia, the tests are much more useful in ruling out $\mathrm{AD}$ as the cause of symptoms than ruling it in. New data that will emerge over the next few years will likely improve specificity by using models that can include more sensitive cognitive tests, genetic risks, and trajectories of change over (hopefully) very short time periods (Koychev et al., 2017). From these reviews (e.g. Ritchie et al., 2014) it was possible to calculate clinically meaningful incremental values in diagnosis using likelihood ratios, which confirmed there was much greater benefit of negative tests ruling out the possibility of decline to dementia compared to positive tests predicting decline. This creates a complex dynamic between the clinician and the patient where it is vital the patient receives accurate information of the value and inherent uncertainty of the test and highlights the incredible value in the form of follow-up appointments to undertake repeat testing. Moreover, the intricacies and complex interpretation of these test findings are explained by highly experienced specialists who, themselves, are comfortable interpreting the biomarker results in a wider neurodegenerative disease context. An imprecise understanding of risk of conversion to dementia for a patient could lead to psychological harm and distress that may itself impact negatively on the neurodegenerative disease process. The harm from poor disclosure of test results is a major research focus currently in parallel to the more fundamental understating of the patho-clinical value of the biomarkers.

\section{The future of $\mathrm{MCl}$ incorporates biomarkers as well as risk factor identification}

AD probably starts developing decades before clinical dementia when the person is cognitively asymptomatic. Thus, the current approach to neurodegenerative disease research is focusing on a younger asymptomatic at-risk group using biomarker evidence and sensitive cognitive testing. A recent review of innovation in the field of dementia (Dementia Innovation Readiness Index; Global Coalition on Aging and Alzheimer's Disease International, 2017) identified prevention and risk reduction as a key theme in solving the growing global dementia problem. Better case identification would lead to interventions being developed in the most appropriate sample group and meaningful and relevant findings of stopping progression of either healthy or mildly impaired individuals in developing Alzheimer's dementia. We know from $\mathrm{AD}$ pathology that by the time any cognitive impairment becomes evident, neurofibrillary tangles will have spread widely in the cortex with spread possibly being triggered by the generation of amyloid- $\beta$ plaques (Sacuiu, 2016). Identifying the sequence and interactions between biological changes at a systems level is an important and surmountable challenge and one which must also incorporate measures of other relevant disease processes such as inflammation and cerebrovascular disease. While biomarker testing is important in clinical trials to exclude people without $\mathrm{AD}$ pathology, there nevertheless remains a high probability that people who test positive do not progress to dementia. Therefore, the next phase of diagnostic criteria development will be to delineate the different stages of neurodegeneration combining cognitive tests designed to detect a signal at a much earlier stage in very specific brain areas, notably entorhinal cortex, posterior hippocampus and precuneus, and also 
incorporating risk factors in the disease modeling (e.g. age or $A P O E$ status), more sensitive measures of hippocampal function and a sense or measure of intra-individual change between at least two but ideally three time points. Hence, developing the four-dimensional model referred to earlier.

\section{Clinical trials involving a younger preclinical population}

After decades of research, there are still no diseasemodifying pharmacological interventions for $\mathrm{AD}$. While targeting the prodromal period to prevent dementia from manifesting is the logical direction of developing treatments, testing such treatments on an inappropriate sample (e.g. false positive) would lead to inaccurate findings. In order to minimize the risk of this occurring, new AD studies focus on younger healthy volunteers from as early as mid-life to collect longitudinal data on risk factors and investigate very early pathologies. Around 35\% of dementia is proposed to be attributable to a combination of the following nine risk factors: low educational attainment; mid-life hypertension and obesity; hearing loss; late-life depression; diabetes, physical inactivity, smoking, and social isolation (Livingston et al., 2017). The nuance is detecting pathological changes early, using biomarker assessments, detailed neuroimaging, and neuropsychological tests. This may lead in the first instance to advice on risk factor modification and if a breakthrough occurs in disease understanding, a pharmacological intervention (or combination) would be initiated. This is the approach taken in the European Prevention of Alzheimer's Dementia project (EPAD; Ritchie et al., 2016) and the Global Alzheimer's Platform (GAP; Cummings et al., 2016). These major and aligned international programs are based on the understanding that dementia is the final stage of an illness that starts developing far earlier when no symptoms are expressed.

\section{A need for modern statistical tools driving precision medicine}

To detect early pathological changes, it is necessary to track change in younger individuals using sensitive tools and adequate study designs. Although more research is needed to identify the optimal study design to accurately capture the onset of decline, frequent data collections are necessary to avoid missing such onsets. But new data collection designs implemented in novel research programs, must also be accompanied by the use and development of adequate analytical tools to capture the multi-dimensional dynamics of the various operating processes, which are not accounted for in commonly-used prediction models such as logistic regression or Cox proportional hazards models. Improved statistical models exist but are not yet commonly used. For instance, multivariate joint longitudinal survival models represent an effective tool that fulfills several of the desired features of these needed new risk prediction models (Hickey et al., 2016). Furthermore, models need to capture and better explain heterogeneity between individuals that we observe in clinical practice, acknowledging the existence of groups with diverse trajectories of change (Proust-Lima et al., 2007). Ignoring this heterogeneity may simply mask individuals at higher risk of disease, or fail to identify the group-specific role of certain risk factors. Embracing heterogeneity is a prerequisite if we are to attain precision medicine paradigms that recognize the importance of individualized drivers of disease, trajectories of decline, and therein precise interventions that are tailored to that person (Hampel et al., 2016).

\section{Conclusions}

The future evolution of the concept of MCI sees the condition in fact become extinct. If we can deliver on the promise of better characterizing the clinical spectrum of neurodegenerative disease that relies on (1) multi-domain biomarkers and more accurate neuroimaging techniques looking at a much more detailed part of the brain complemented with (2) more sensitive and psychometrically robust computerized neuropsychological testing, and (3) risk factors both fixed and modifiable with (4) good measures of change, then large categorical approaches will be replaced by accurate individualized predictions. This approach will not only identify the disease accurately to create an appropriately phenotyped sample for clinical trials but also better distinguish neurodegeneration from other neuropsychiatric conditions like anxiety and depression and help drive personalized therapeutic and prevention interventions. The cohorts are unlikely to yield applicable algorithms for several years. Until then, clinical practice remains somewhat dependent on often quite subjective clinical judgments in assessing MCI. The emerging reliance on biomarkers needs to be encouraged but clinicians must be cautious about over-valuing their utility in improving accuracy given quite small (positive and negative) likelihood ratios and the complex communication of uncertain test results to patients. For now, the main reason for identifying 
MCI or any other prodromal phase of dementia could well be to help engage people in clinical research and offer pragmatic advice on life style and health modifications, which undoubtedly have a beneficial effect on brain health.

\section{Conflict of interest}

The research leading to these results has received support from the Innovative Medicines Initiative Joint Undertaking under grant agreement no. 115736, resources of which are composed of financial contribution from the European Union's Seventh Framework Programme (FP7/2007-2013) and EFPIA companies' in kind contribution.

\section{Stina SAunders, ${ }^{1}$ Karen Ritchie, ${ }^{1}$ TOM C. Russ, ${ }^{1,2}$ Graciela Muniz-Terrera ${ }^{1}$ and Craig W. Ritchie ${ }^{1}$}

${ }^{I}$ Centre for Dementia Prevention, Centre for Clinical Brain Sciences, University of Edinburgh, Edinburgh, $\mathrm{UK}$

${ }^{2}$ Alzheimer Scotland Dementia Research Centre, University of Edinburgh, Edinburgh, UK

\section{References}

Cummings, J. et al. (2016). Re-engineering Alzheimer clinical trials: global Alzheimer's platform network. The Fournal of Prevention of Alzheimer's Disease , 3, 114120.

Hampel, H. et al. (2016). Precision medicine - the golden gate for detection, treatment and prevention of Alzheimer's disease. The fournal of Prevention of Alzheimer's Disease, 3, 243-259.

Heinik, J. (2006). V. A. Kral, the Montreal Hebrew old people's home, and benign senescent forgetfulness. History of Psychiatry, 17 (3), 313-332

Hickey, G. L., Philipson, P., Jorgensen, A. and Kolamunnage-Dona, R. (2016). Joint modelling of time-to-event and multivariate longitudinal outcomes: recent developments and issues. BMC Medical Research Methodology, 16, 117.

Kogan, M. and Jeong, H. S. (2017). Alzheimer's disease. In D. Rakel (ed.), Integrative Medicine, 4th edn, Philadelphia, PA: Saunders Elsevier.

Koychev, I. et al. (2017). Deep and frequent phenotyping study team. PET Tau and Amyloid- $\beta$ burden in mild Alzheimer's disease: divergent relationship with age, cognition, and cerebrospinal fluid biomarkers. The Fournal of Prevention of Alzheimer's Disease, 60, 283-293.
Livingston, et al. (2017). Dementia prevention, intervention, and care. The Lancet Commissions. Epub ahead of print, doi:10.1016/S0140-6736(17)31363-6.

Olsson, B. et al. (2016). CSF and blood biomarkers for the diagnosis of Alzheimer's disease: a systematic review and meta-analysis. Lancet Neurology, 15, 673-684.

Proust-Lima, C., Letenneur, L. and Jacqmin-Gadda, $\mathbf{H}$. (2007). A nonlinear latent class model for joint analysis of multivariate longitudinal data and a binary outcome. Statistics in Medicine, 26, 2229-2245.

Ritchie, K. and Ritchie, C. W. (2012). Mild cognitive impairment (MCI) twenty years on. International Psychogeriatrics, 24, (1), 1-5.

Ritchie, C. et al. (2014). Plasma and cerebrospinal fluid amyloid beta for the diagnosis of Alzheimer's disease dementia and other dementias in people with mild cognitive impairment (MCI). The Cochrane Database of Systematic Reviews, 10, 1-90.

Ritchie, C. W., Molinuevo, J. L., Truyen, L., Satlin, A., Van der Geyten, S. and Lovestone, S. (2016).

Development of interventions for the secondary prevention of Alzheimer's dementia: the European prevention of Alzheimer's dementia (EPAD) project. The Lancet Psychiatry, 3, 179-186. doi:10.1016/s2215-0366(15)00454-x

Ritchie, C. W., Smailagic, N., Noel-Storr, A. H., Ukoumunne, O., Ladds, E. C. and Martin, S. (2017). CSF tau and the CSF tau/ABeta ratio for the diagnosis of Alzheimer's disease dementia and other dementias in people with mild cognitive impairment (MCI). The Cochrane Database of Systematic Reviews, (3), 1-102.

Sacuiu, S. F. (2016). Chapter 8 - Dementias. In C. Rosano, M. A. Ikram, and M. Ganguli (Eds.), Handbook of Clinical Neurology. Neuroepidemiology, vol. 138 (pp. 123-151). Amsterdam: Elsevier.

Smailagic, N., Vacante, M., Hyde, C., Martin, S., Ukoumunne, O. and Sachpekidis, C. (2015). (1)(8)F-FDG PET for the early diagnosis of Alzheimer's disease dementia and other dementias in people with mild cognitive impairment (MCI). The Cochrane Database Systematic Reviews, 1, 1-105. doi:10.1002/14651858.CD010632.pub2

Sperling, R. A. et al. (2011). Toward defining the preclinical stages of Alzheimer's disease: recommendations from the National Institute on Aging-Alzheimer's Association workgroups on diagnostic guidelines for Alzheimer's disease. Alzheimer's and Dementia, 7, 280-292. doi:10.1016/j.jalz.2011.03.003.

Zhang, S. et al. (2014). (11)CPIB-PET for the early diagnosis of Alzheimer's disease dementia and other dementias in people with mild cognitive impairment (MCI). The Cochrane Database Systematic Reviews, 7, 1-105.

Global Coalition on Aging, Alzheimer's Disease International. (2017). Dementia Innovation Readiness Index. Available at: http://www.globalcoalitiononaging.com/ data/uploads/documents/gcoa-adi-dementia-index.pdf; last acessed 11 September 2017. 